\title{
Végétaux ligneux et pâturages des savanes de l'Adamaoua au Cameroun
}

\author{
par J. PIOT
}

\begin{abstract}
RÉSUMÉ
De graves problèmes d'envahissements ligneux sur les pâturages de l'Adamaoua en général rendent d'actualité cette étude des savanes arbustives et arborées qui couvrent le plateau.

L'auteur étudie trois aspects essentiels de cette vaste question.

10 action des feux sur la végétation ligneuse ;

$2^{\circ}$ rôle de l'Intensité de la charge en bétail sur cette même végétation ; 30 valeur pastorale des éléments ligneux des terrains de parcours.

Des conclusions sont données visant à définir une politique des feux en matière forestière, et surtout pastorale ef également sont proposées des bases d'aménagement sylvo-pastoral dans l'optique de l'ınévitable évolution de l'élevage vers des formes moins extensives.
\end{abstract}

Si les savanes africaines sont revendiquées à la fois par les forestiers et les éleveurs en ce qui concerne la gestion de leur exploitation extensive, c'est bien parce que leur vocation est souvent difficile à définir. Suivant le type de savane considéré et son utilisatıon, l'équilibre herbe-ligneux peut se déplacer dans un sens ou dans l'autre. La Station Fourragère de Wakwa a eu pour but dès sa création (1956) d'étudier les actions du parcours et des feux sur la savane.

La présente étude décrit essentiellement le comportement du matériel ligneux : l'évolution de la végétation herbacée fera l'objet de communications ultérieures.

L'arbre et l'arbuste procurent aux animaux, en période difficile surtout, une partie de leur ration; i il était intéressant d'essayer de la préciser qualitativement et quantitativement.

\section{A) CONDITIONS NATURELLES DE L'ADAMAOUA}

L'Adamaoua est un vaste plateau d'une altitude moyenne de 1.000 à $1.200 \mathrm{~m}$, situé entre les $6^{\mathrm{e}}$ ef $8 \mathrm{e}$ degrés de latitude Nord (6) (8).
Le climat est du type Soudano-Guinéen montagnard avec $1.600 \mathrm{~mm}$ de pluies en 8 mols, et 4-5 mois écologiquement secs.

La température moyenne annuelle est de $23^{\circ}$ (maximum et minimum absolus respectivement de $35^{\circ}$ en mars et $10^{\circ}$ en janvier). Les moyennes mensuelles sont de $32^{\circ}$ en mars pour les maximums et $13^{\circ}$ en décembre et janvier pour les minimums.

L'humidité relative moyenne est de 75 p. 100 en saison des pluies, 40 p. 100 en saison sèche, et passe par des minimums absolus de l'ordre de 10 p. 100 en février, ce qui a de sérieuses conséquences écologiques.

Géologiquement, l'Adamaoua est constitué par un socle granitique ef gneissique avec des placages de grès et surtout de roches volcaniques, basaltiques le plus souvent.

Les sols formés sur ces derniers types de roche mère sont en général nettement plus riches que les qutres, surfout sur les derniers épanchements basaltiques qui donnent naissance à un sol foncé peu évolué. Très entaillé par les vallées, parsemé de zones d'épandages des cours d'eau ou de marais, l'Adamaoua possède d'assez vastes 
surfaces de sols hydromorphes précieux pour le parcours des troupeaux en salson sèche.

La végétation qui recouvre la plus grande partie du plateau est une formation mixte forêtgraminée de type savane arbustive et arborée à Daniellia et Lophira.

La liste des autres espèces ligneuses qu'on y rencontre et qui comporte une soixantaine d'espèces est donnée en annexe.

Les vallées et les thalwegs sont occupés en grande partie par des galeries forestières dont la flore ligneuse est très riche, les espèces les plus fréquentes étant:

\author{
Neoboutonia velutina. \\ Parmari kerstingii. \\ Sterculia tragacantha. \\ Syzygium gumeense D, C. guineense. \\ Tricalysia okelensis var oblanceolata. \\ Uapaca togoensis. \\ Vitex doniana.
}

Une lıste relativement complète des espèces est donnée en annexe.

La végétation herbacée est essentiellement à base d'Hyparrhenia spp avec différents faciès liés à la nature du sol (Hyporrhenla diplandra et Paspalum orbiculare ou Hyparhenia filipendula et Loudetia kagerensis, etc...) ou au type d'exploitation (Panicum phragmitoides, Sporobolus pyramidalis, Andropogon gayanus, Brachioria brizantha avec Hyparrhenia rufa, Hyparrhenia chrysargyrea par exemple).

C'est dans ce contexte naturel qu'au moment de la création de la Station Fourragère (1956-57) un programme d'étude d'action des feux et du parcours sur la végétation a été établi ; ce programme est largement exécuté.

\section{B) EXPÉRIMENTATION ET OBSERVATIONS}

La Statıon de Wakwa située à $1.200 \mathrm{~m}$ d'altitude, 7017 de latitude Nord ef 13033 de longitude Est est un échantillon représentatif des sols et de la végétation de l'Adamaoua.

On y rencontre des sols : (voir carte)

- Granitiques pauvres et à faible rétention superficielle pour l'eau.

Les arènés granitiques de bas de pente sont toutefois plus riches.
- Basaltiques rouges ; formés sur basaltes anciens donc relativement évolués et lessivés.

- Basaltiques foncés ; formés sur basaltes récents, Ils sont peu évolués et riches. Ils forment les meilleurs pâturages de l'Adamaoua.

Les observations sur la végétation ont été faites dans 17 parcs non dessouchés, qui on été soumıs à des traitements différents faisant intervenir les feux ef la charge en bétail. Aınsi, avec les sols ce sont les actions de 3 facteurs de variation qui sont analysées.

\section{MÉTHODOLOGIE}

L'inventaıre de la végétation ligneuse imposaıt un échantillonnage tellement large que, compte tenu de la surface limitée des parcs, il devenait aussi rapide et plus efficace de tout inventorier.

Dans les comptages on distingue les rejets et arbustes $(0$ à $1,50 \mathrm{~m})$ des arbrisseaux $(1,50 \mathrm{~m}$ à $3 \mathrm{~m}$ ) et des arbres (plus de $3 \mathrm{~m}$ ). En falt, des facteurs subjectifs d'appréciation de la part des agents chargés des inventaıres Imposent quelques corrections aux résultats bruts du comptage. En particulier pour certains, l'arbre est encore arbrisseau et l'arbuste ne l'est plus et il est difficile d'agir sur ces notions rendues de faif fort subjectives par la varıété de porf des ligneux. Le comptage des rejets est le plus délicat; les uns recensent absolument tous les brins, alors que les autres opèrent presque par cépées. Mais toutes ces hypothèques peuvent en fait être assez facilement levées. Par contre, en ce qui concerne la désignation spécifique des éléments ligneux, nous considérons les possibilités d'erreur comme très faibles ef lımitées aux lapsus lınguae ou calami, car les plantes sont parfaitement connues des agents.

Les charges de bétail à l'hectare sont chiffrées en $\mathrm{kg}$ de poids vif à l'hectare et la productivité des pâturages en unités fourragères.

\section{CONDITIONS EXPÉRIMENTALES}

Les facteurs de variation sont les suivants :

- Ceux dus au milieu lui-même : les sols.

- Ceux dus à l'intervention de l'expérimentation : 


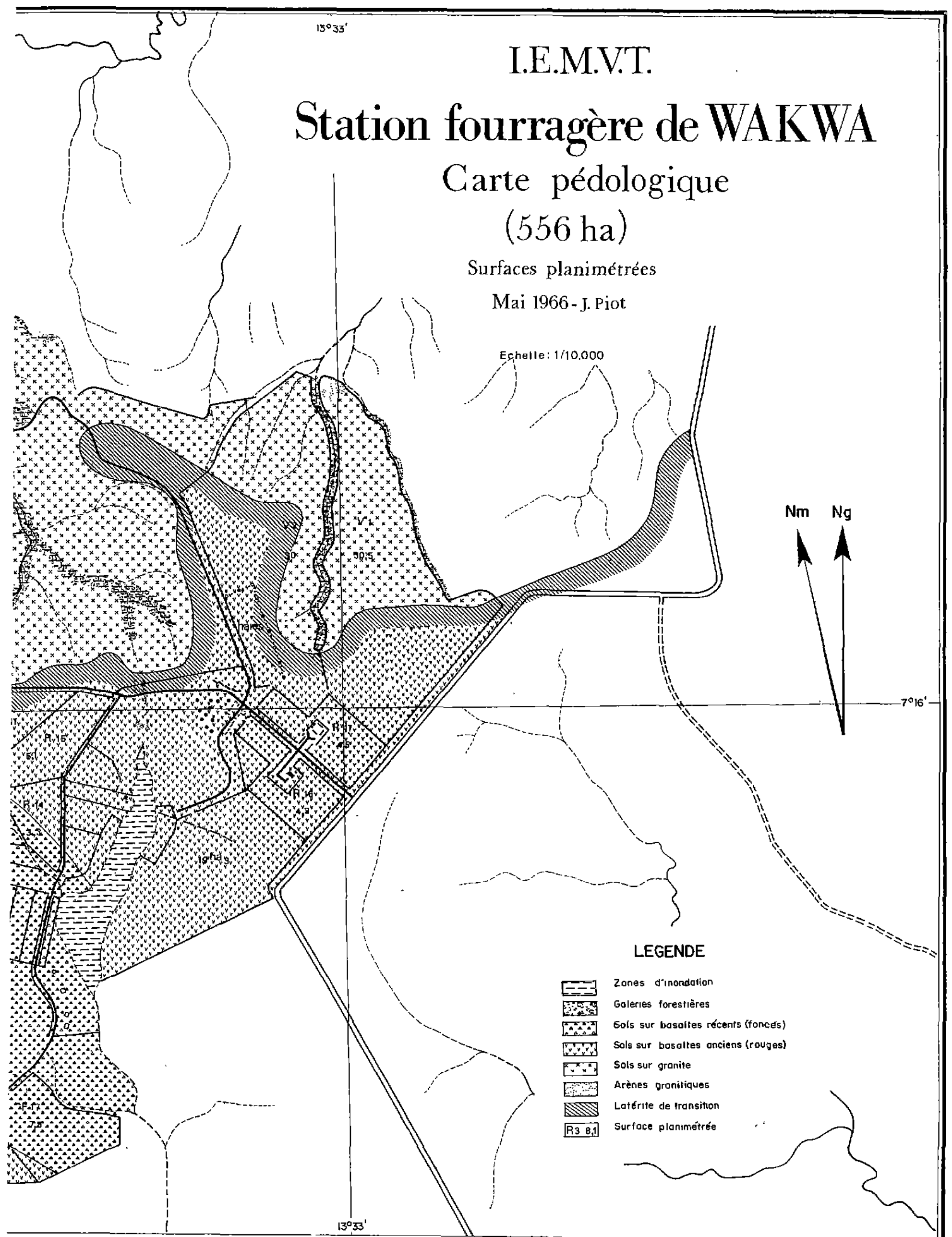


- Charges en bétail.

- Nature des feux soit :

absence de feux, feux précoces,

feux de pleine saison sèche,

feux dits de contre-saison.

\section{CHARGES}

De 1957 à 1962, les charges appliquées aux différents parcs étaient classées en légères, normales, excessıves, sulvant l'évolution ultérieure des caractéristiques de la végétation et du pâturage.

Les animaux séjournaient en permanence nuit et jour chaque année dans les parcs, de mai à décembre, sauf dans 3 parcs, une saison des pluies sur 3.

De 1962 à 1968 les différents traitements ont été les suivants :

En saison des pluies, le cycle d'utılisation des parcs en rotation par les animaux est de 28 jours environ, la durée de la pâture variant suivant les parcs mals étant si possible au plus égale à 8 jours.

Deux types de charge sont prévus $500 \mathrm{~kg} / \mathrm{ha}$ et $250 \mathrm{~kg} / \mathrm{ha}$.

En saison sèche la charge est amenée à $125 \mathrm{~kg} / \mathrm{ha}$ sans rotation.

Certains parcs sont utilisés tous les ans, d'autres mis hors pâture pendant 1 an sur 2, 1 an sur 3 ou 4 ans sur 4 i cette dernière condition étant très semblable à celle des zones de transhumances traditionnelles.

\section{FEUX}

Les types de feux expérimentés sont les suivants :

10 Une absence de feu permanente.

$2^{\circ}$ Des feux précoces ; ils doivent être définis car les concepts du forestier et de l'éleveur sont quelque peu différents.

Le forestier attend du feu précoce l'élimination, le plus tôt possible, d'un matériel végétal qui serait dangereusement combustible en pleın état de siccité pour la végétation ligneuse associée. De là naît une notion de seuil de combustibilité qui est fondamentale : les seuils étant différents suivant les faciès graminéens et le relief. On peut donc être amené à brôler plusieurs jours de suite dans un même secteur, mais le feu ainsi obtenu est alors aussi peu violent que possible.

Par contre, pour l'éleveur, ce feu doit réduire le couvert arboré et arbustif, mals surtout permettre la repousse et l'exploitation du regaın donné par les graminées cespiteuses pérennes. Plus le feu est précoce et plus la repousse sera abondante car elle profite d'une humidité du sol qui ne fera que baisser avec le temps.

30 Des feux de pleine soison sèche :

On attend d'eux un maximum de dommages aux végétaux ligneux et si possible un peu de regain, ce qui pose peu de problèmes jusque fin janvier (sauf en terraın granitıque à faible rétention pour l'eau). On veut aussi dans la mesure du possible, éviter de brôler les jeunes feuilles des espèces appelées qui débourrent (Daniellha par exemple dès mi-janvier).

40 Enfin des feux dits de contre-saison :

Ce sont des feux allumés dès le retour des pluies et essentiellement pour nettoyer les pâturages des refus qui peuvent les encombrer, mais que le bétail aura pu exploıter en partie pendant toute la salson sèche. Expérimentalement, on veut voir si ce feu n'est pas plus efficace contre l'envahissement ligneux que les autres feux, puisqu'il les touche en pleine montée de sève (cette dernière commence 3 ou 4 semaines avant le retour des pluies).

Utilisation des feux.

De 1957 à 1962, les parcs étaient brôlés tous les ans à l'un des régimes ci-dessus et cela quelle que soit la densıté du couvert herbeux existant laissé par les animaux juste avant la mise à feux.

A partir de 1962 compte tenu des observations déjà faites et l'emploi systématique de la rotation pour exploiter le pâturage, le régime des feux est le suivant :

Dans la série des essais où le cycle d'utilisation est de 4 ans les parcs sont brûlés 3 ans de suite soit précocement, solt en pleine saison sèche, soit en contre-saison ; d'autres sont brûlés 2 années de suite en contre-saison (début de saison des pluies) et la $3^{\mathbf{e}}$ année en pleine saison sèche.

Ces parcs sont utilisés comme pâturage la 


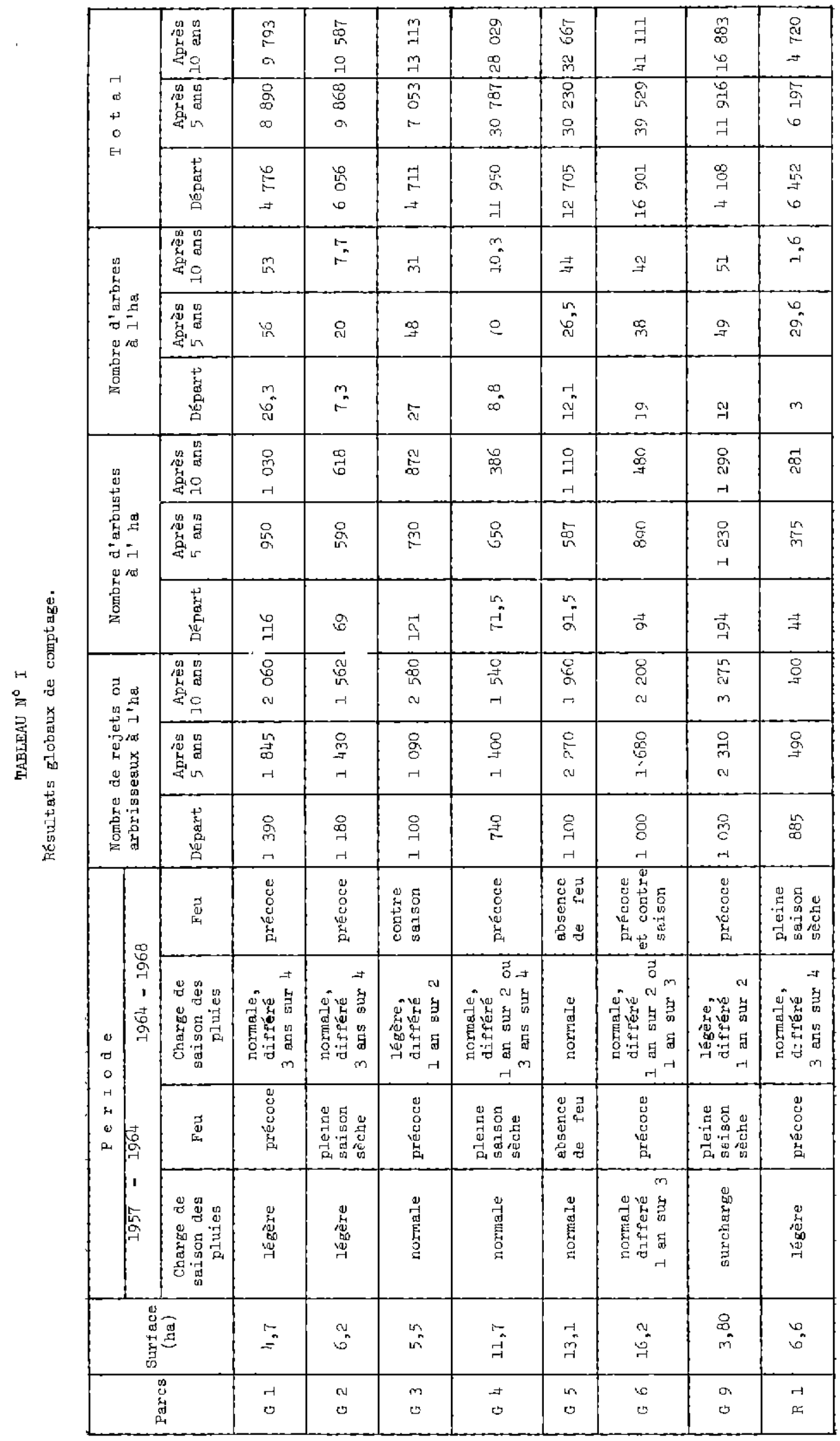




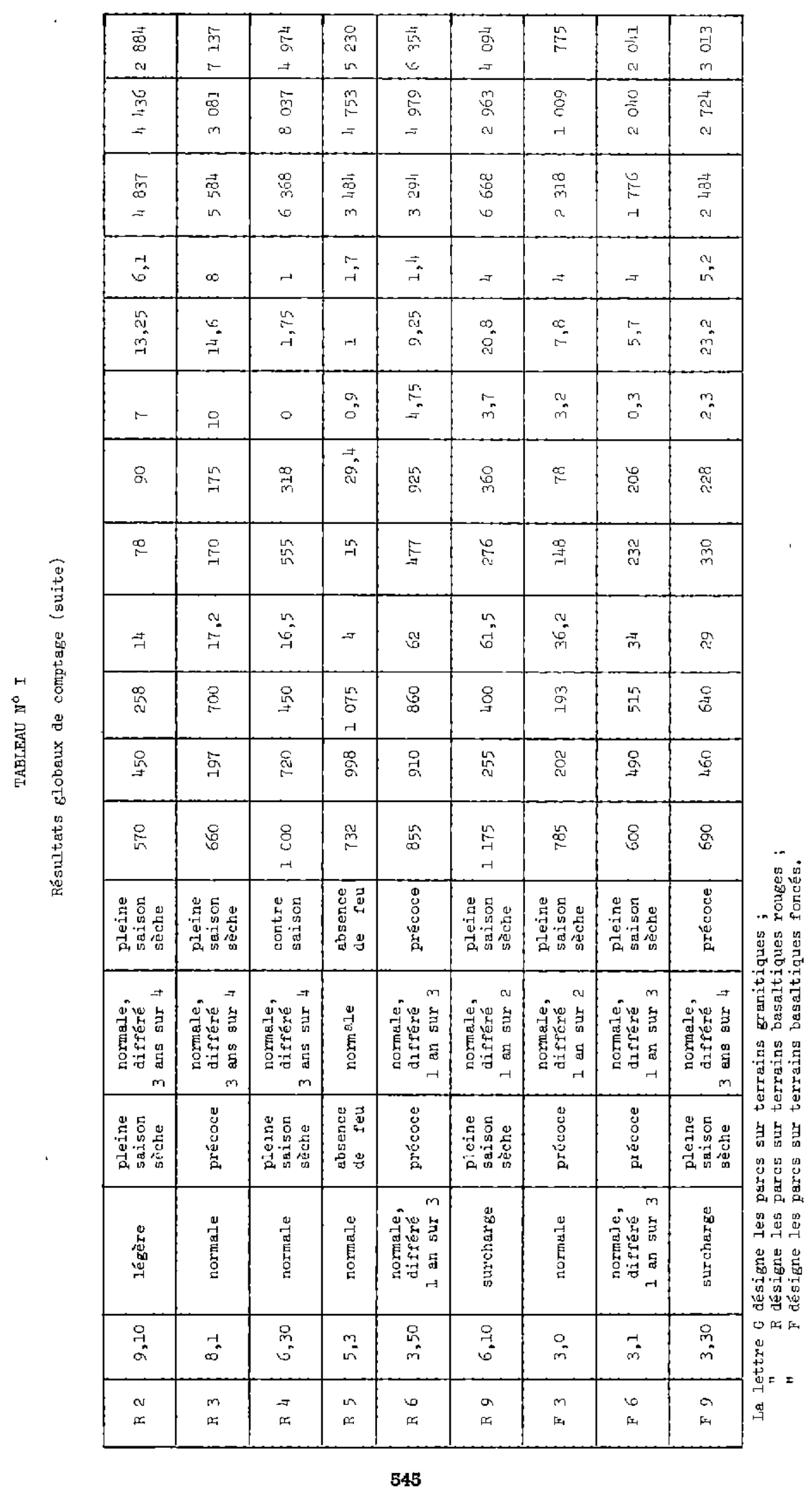


quatrième année quels que soient les traitements effectués par le feu.

Dans les autres séries où le cycle est de 2 ou 3 ans, les mêmes types de feux sont appliqués comme précédemment après la ou les saisons des pluies qui suivent celle pendant laquelle le parc a été pâturé.

Le protocole d'emploı des feux a été modifié en 1963 en rasson du falt que la qualité du pâturage allait en diminuant alors que ces recherches visaient d̀ définir un mode d'explostation rationnel du pâturage.

\section{C) RÉSULTATS}

L'expérimentation a commencé en 1957-58 par un comptage général des ligneux dans les 17 parcs. Un second comptage a été effectué en 1962-63 dont les résultats ont été en partie analysés (6). Enfin un troisième a eu lieu en 1967-68.

Le tableau no 1 résume l'ensemble des traitements et les résultats globaux des comptages.

$$
\text { ** } *
$$

\section{a) Analyse en fonction des sols.}

Les inventaires montrent que d'une manière générale, les traitements imposés mènent d̀ un accrolssement du matériel ligneux beaucoup plus important en terrains granitiques qu'en terrains basaltiques rouges, et en ces derniers, plus qu'en terrains basaltiques foncés.

L'embuissonnement parâ̂t donc d'autant plus fort que le terrain est pauvre. Pour les 2 types basaltiques, situés dans un site tout à fait comparable, l'explication peut tenir au fait que les charges supportables sont effectivement plus élevées et, qu'en outre, lorsque les feux passent, ils sont plus violents dans les parcs riches parce qu'alimentés par un matériel combustible plus important.

\section{b) Analyse en fonction des traitements.}

10 Action du pâturage intensif sans feu :

- En terrain granitique - Etude du Parc G5. Dans les 5 premières années, il est constaté un développement extraordinaire des rejets et arbustes.
Les 5 années sulvantes on constate une régressıon logique à ce niveau d'encombrement des rejets et jeunes plants, alors que le changement de classe d'une bonne partie d'entre eux vient presque doubler l'effectif des arbustes.

Le phénomène est le même pour les arbres et l'ensemble contribue, en fermant le couvert, à donner, plus que le comptage, une impression puissante d'envahissement.

- En terrain basaltique - Ełude du Parc R5.

On est parti en général pour les parcs basaltiques d'une couverture arbustive plus modeste. Ces sols plus riches ont été plus ou moins cultivés dans le passé, donc défrichés, et l'ambiance de départ y était déjà beaucoup moins forestière.

On constate donc après 10 ans une poursuite de l'évolution qui n'a guère duré que 5 ans en granitique.

20 Action d'une charge excessive avec feu de pleine saison sèche.

Le comportement de ces parcs est très important à considérer car il correspond aux conditions quasi générales d'exploitation actuelle des terrains de parcours de l'Adamaova : charge annuelle maximale, feu annuel (plus ou moins tardıf, mais cela est de peu d'importance, car ces feux alimentés par une herbe courte causent très peu de dommages aux arbres).

- En terrain granitique - Etude du parc G9.

L'évolution est très comparable à celle du parc non brûlé homologue (G5) avec une poursuite de la progression des rejets (Horungana madagascariensis surtout). On tend là aussi vers une fermeture complète du couvert avec disparition de l'herbe. Alors que nous avons toujours $500 \mathrm{~kg} / \mathrm{ha}$ en G5, G9 est presque surchargé avec $250 \mathrm{~kg} / \mathrm{ha}$.

Donc, avec peu de bétail et des feux peu importants, on constate une évolution comparable à celle des séries non brôlées, ce qui est normal.

- En terrain basaltique rouge - Etude du parc R9.

L'inventaire de ce parc nous semblait aberrant en 1963, et nous avons pensé à l'époque que des exploitations délictueuses avaient pu être pratıquées. Les 5 dernières années montrent une évolution comparable à celle de $\mathrm{G} 9$ avec beaucoup moins d'ampleur. 
- En terrain basaltıque foncé - Etude du parc F9.

L'augmentation du couvert ligneux tatal est, là aussi, assez importante malgré, en 1963, un inventaire douteux des rejets et des arbustes. Nous sommes en tout cas dans les conditions d'envahissement ligneux presque aussi importantes que dans les parcs non brûlés et chargés tous les ans. A plus ou moins longue échéance, cela conduira à un étouffement grave du tapis herbacé en ce qui concerne la productivité en salson des pluies des pâturages. C'est d'ailleurs, hélas, le phénomène que l'on constate en de nombreux secteurs de l'Adamaova.

$3^{0}$ Action des feux de pleıne saison sèche:

Etude comparative des parcs granitiques G2-G4 et basaltiques rouges R2-R4.

Ils montrent typiquement un moindre développement, voire une régression du couvert ligneux.

Il est assez difficile de faire intervenir la notion de charge dans la dernière période quinquennale, car tous ces parcs ont été depuis 1965, soit différés, soit chargés à $500 \mathrm{~kg} / \mathrm{ha}$.

Pourtant, bien qu'une charge faible puisse avoir comme conséquence un feu mieux alimenté par plus de refus, il semble bien que c'est à une charge supérieure qu'est due la régression constatée de G2 à G4 d'une part et de R2 à R4 d'autre part.

Le parc G2 a subi des feux précoces en 1965 et 1966 après mise en différé, ce qui explique peut-être la faible augmentation du matériel ligneux.

40 Action des feux précoces sans mise en différé.

Etude comparative des parcs granitiques : G1-G2-basaltiques rouges R1-R3 et basaltiques foncés F3.

L'augmentation de l'embuissonnement pour G3 ef $\mathrm{R} 3$ est très nette (bien que l'on puisse, pour ce dernier parc, douter de la valeur des chiffres de 1963). G1 se maintient à 10 p. 100 près.

Par contre, R1 et F3 s'éclaircissent. R1 (comme $R 2$ et R4) a. été sous-chargé en 63 et 64 , et a subi après mise en différé un feu de pleine saison sèche en 1966. F3 sous-chargé également en 63 et 64 a eu un feu de contre-saison en avril 66.
Ces deux derniers parcs ont donc en fait été parcourus 3 ans de suite par des feux qui ont dû être assez violents et responsables des diminutions constatées.

50 Action des feux précoces avec mise en différé des pares : Etude comparative des 3 types de parcs $G 6, R 6$ et $F 6$.

$\mathrm{G} 6$ et $\mathrm{F} 6$ maintiennent à peu près leur taux d'embuissonnement alors que R6 accroît son total de ligneux de près de 30 p. 100 . Une année de mise en différé sur trois est sûrement suffisante, associée d̀ un feu précoce (et probablement aussi à un feu de pleine saison sèche) pour farre régresser l'embuissonnement ou en tout cas le contenir.

Quel est des deux facteurs, charge et feu, celui qui a le plus d'importance? Nous ne pouvons encore y répondre très sûrement, mais inclinons vers une prépondérance de l'influence de la charge. Dans les parcs ou l'embuissonnement a diminué, ce ne sont pas les espèces appétées qui ont plus partıculièrement régressé : donc, l'influence de la charge est certainement plus liée aux piéfinements et aux passages du bétaif qu'à la pâture elle-même. Par contre, les Harungana madagascoriensis, non appétés, sensibles au feu se sont installés même dans ces parcs sous forme de rejets, ce qui ne se serait pas produit avec des feux assez importants.

Quant aux terrains granitiques, ils sont pauvres (productivité de l'ardre de 1,250 U. F./ha) et ne supportent donc qu'une charge assez faible. La végétation herbacée $y$ est en grande partie constituée de Loudetia (type kagerensis) et d'autres graminées annuelles fines, aliments pour un feu très fugace. Le relief $y$ est particulıèrement accentué, ce qui permet aux feux de passer très rapidement des bas-fonds où on les allume jusqu'aux sommets. Or, les feux trop rapides (qui montent les pentes ou qui sont poussés par un vent trop fort) brûlent très mal les grosses palles et abîment peu les arbres. D'autre part, ces sols granitiques sont sıllonnés de galeries qui hébergent des espèces plus ou moins forestières toutes prêtes à «sauter dans la savane», et à la repeupler dès que les conditions le permettent. On trouve en terraın granitıque une variété beaucoup plus grande d'espèces que dans les autres, et en particulier d'espèces apparues dans les 10 dernières années. Enfin, les 
sols granitiques sont en eux-mêmes sûrement plus favorables d̀ l'installation des systèmes racinarres des arbres que les sols plus ou moins compacts et vite secs des basaltes. L'allure assez chaotique et crevassée des zones granitiques crée constamment des sites favorables à l'accrochage de la végétation arbustive.

Toutes ces ralsons sont susceptibles d'étayer une explication de ces différences de comportement en terrains granitiques et basaltiques qui apparaissent incontestablement. Mais II nous manque encore quelques années d'expérimentation pour pouvoir hiérarchiser ces différents facteurs.

\section{c) Evolution spécifique.}

Les observations faites sur les principales espèces dans les différents parcs classés en fonction de la nature du terrain ont été rassemblées dans les tableaux II et II bis en vue de comparer les résultats obtenus dans les domaines

TABLEAU NT II $^{\circ}$

Rang de classement relatif des espèces ligneuseg

\begin{tabular}{|c|c|c|c|c|c|c|c|}
\hline & \multirow{2}{*}{ Esp } & \multicolumn{3}{|c|}{ Basgltiques rouges } & \multicolumn{3}{|c|}{ Basaltiques foncés } \\
\hline & & 1958 & i963 & 1968 & 1958 & 1963 & 1968 \\
\hline & Annona arenaria & 1 & 2 & 1 & 1 & 1 & 1 \\
\hline & Ditiostigma thonningit & 2 & 1 & 2 & 2 & 3 & 3 \\
\hline & Hymenocardia acida & 3 & 3 & 3 & 3 & 2 & 2 \\
\hline & Isorospermum gíaberrimum & 4 & 4 & 4 & 4 & 8 & 8 \\
\hline & Syzygirm guineense macroptera & 5 & 5 & 7 & 9 & 10 & 10 \\
\hline & Psorospermum febrifugum & 6 & 6 & 8 & 6 & 4 & 4 \\
\hline & Bridetia fermiginea & 7 & 7 & 12 & 7 & 7 & 5 \\
\hline & Triohitia roka & 8 & 14 & 11. & & & \\
\hline & Eroted elitiottii & 9 & 13 & 17 & & & \\
\hline & Entada abussinica & 10 & 8 & 10 & 5 & 5 & 7 \\
\hline & Combretum nigricans & 11 & 0 & & & & \\
\hline & Stereospermum kunthianum & 12 & 10 & 15 & 8 & 12 & 16 \\
\hline & Vitex madiensis & 13 & 12 & 20 & 9 & 11 & 15 \\
\hline & Gardenia temifoita & 74 & 15 & 16 & 15 & 15 & 23bis \\
\hline & Enythrina senegatensis & & & & 10 & 13 & 18 \\
\hline & Danieliza oliveni & 15 & 11 & & & 18 & 23 \\
\hline & Jannea schimpen & 16 & 17 & 19 & & & 25 \\
\hline & Securidaca iongepeduncuiata & & & & 12 & 19 & \\
\hline & Terminatia spp. & 17 & 18 & & 11 & 6 & 19 \\
\hline & Albizzia coniaria & & & & 13 & 19 & 21 \\
\hline & Haringana madagascariensis & & 16 & 6 & & & 9 \\
\hline & Aibizia aygia & & & & 1.4 & 21 & 22 \\
\hline ' & Cussonia barteri & & & 5 & & & 20 \\
\hline & AzZophyzus afriaconus & & & 9 & & 16 & 11 \\
\hline & Croton macrostachyus & & & 13 & & 17bis & 12 \\
\hline & Ficus spp. & & & lobis & 12 his & 14 & 14 \\
\hline & Steganotaenia araliacea & & & 14 & & & \\
\hline & Aaesa Zanceolata & & & 18 & & & 13 \\
\hline & Vemonia amygatina & & & & & 9 & 6 \\
\hline & Craterispermum Zawinum & & & & & & 17 \\
\hline
\end{tabular}


basaltiques rouges, basaltiques foncés ef granitiques.

Ces deux tableaux indiquent les principales espèces présentes dans 3 types de terrains par ordre d'importance numérique décroissante au début de l'expérience.

Les classements indiqués ensuite pour 1963 et 1968 permetient de se faire une idée du développement de certaines espèces, compte tenu du fait que, pratiquement, les effectifs de chacune se sont au moins maintenus.
Ces tableaux permettent d'emblée une différenciation botanıque des différentes sortes de terrains. La dominance des espèces reste à peu près celle qui fut sıgnalée en 1964 (6).

Parmi les espèces de première grandeur, les Terminalia sont les seuls à manifester un comportement édaphique certain :

- Terminalia macroptera (et Terminalia dewevrei) caractérise les sols granıtiques et latéritiques par sa présence et son abondance.

- Terminaha glaucescens (et Ormocarpum

TABLEAU $\mathrm{N}^{\circ}$ IIbis

Reng de classement reletif des espèces ligneuses en terrains granitiques

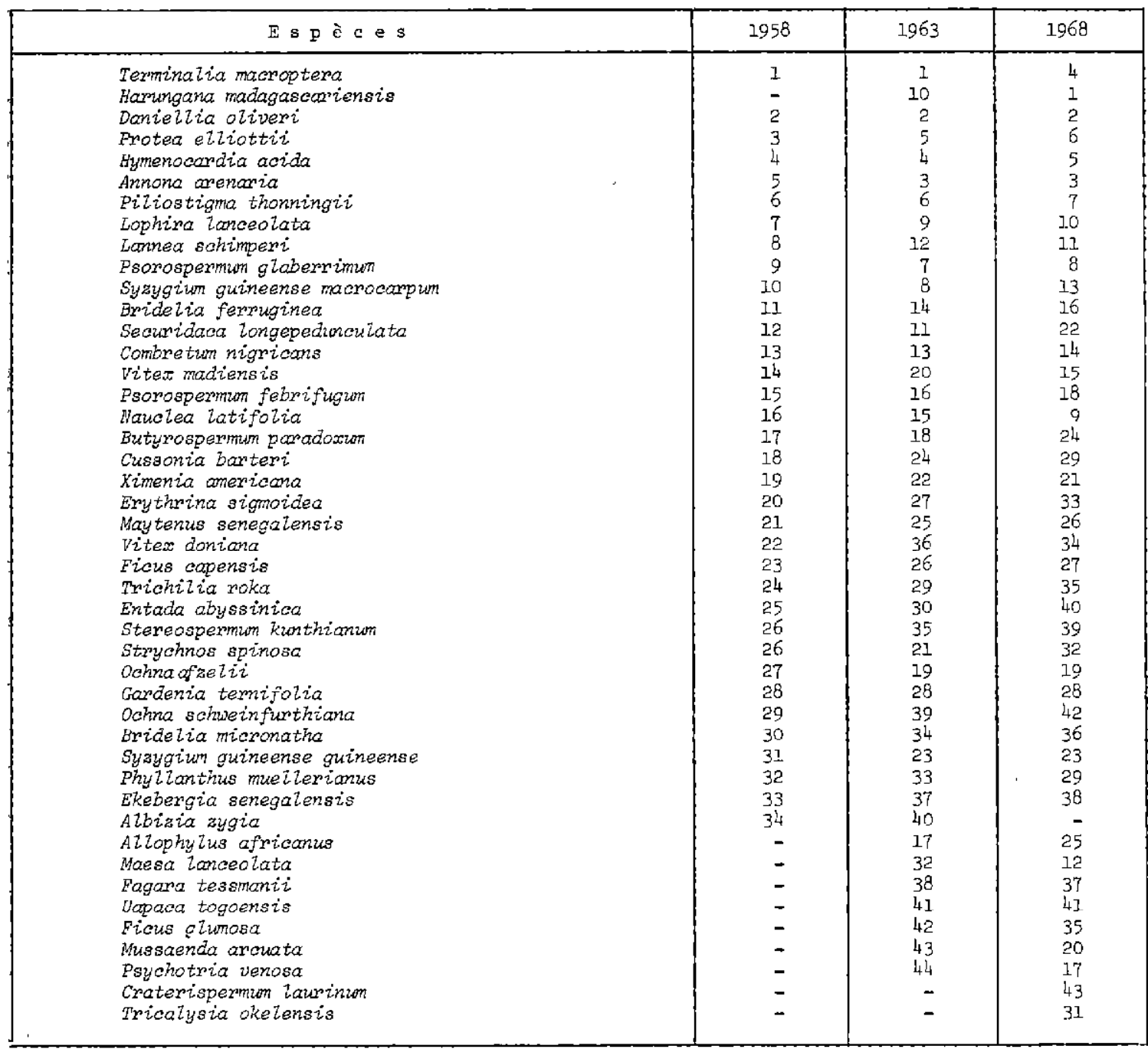


bibracteatum) caractérise les sols basaltiques foncés et se développe de préférence dans les zones à blocs rocheux superficiels.

Toutes les autres espèces dominantes semblent pouvoir se développer aussi bien en sols granitiques que basaltiques.

Par contre, au niveau des espèces secondaires ou rares et simplement présentes, on pourrait trouver en comparant les relevés de nombreuses différences : mais alors l'échantillonnage devient insuffisant en particulıer pour les sols basaltiques.

On ne retiendrait donc guère comme semblant propres aux terrains granitıques que le Faurea speciosa peu fréquent et, molns exclusivement, Lophira lanceolata ef Butyrospermum paradoxum.

Dans ces savanes, ef sans aucun doute, ce sont essentiellement les facteurs feux et eau (galeries, nappes phréatıques, etc...) qui déterminent la répartition des espèces ligneuses, la nature du sol n'intervenant que secondairement. Quant au couvert herbacé, nous avons déjà indiqué (8) que sa composition et sa nature sont déterminées plus par les traitements qu'il subit (feux, intensité du pacage, culture, etc...) que par les conditions édaphiques.

C'est pourquoi dans les conditions d'exploitation de l'élevage extensif africain, il faut attacher beaucoup plus d'importance à la notion de formation qu'à celle d'association trop susceptible de variation, sauf dans des cas limites (sols inondés, dalles latéritıques, etc...).

\section{Evolution des espèces des pâturages non brûlés :}

La série charge correcte sans feux des parcs 5 nous permet de considérer une évolution dans les conditions d'embuissonnement les plus favorables et de loin les plus sôres expérimentalement.

\section{- Terrains granitiques (G5).}

L'élément le plus important d'apport est incontestablement l'Harungana madagascariensis qui se maıntenait jusque-là en bordure de galeries et se multiplie maintenant partout en fourrés denses où l'herbe n'a plus de place, mais où vont par contre venir s'abriter des espèces de forêts (Fagara tessmanii, Psychotria venosa, Syzygium guineense, Mussaenda spp, Hymenodictyon floribundum, Tricalysio okelensis,
Craterispermum lourinum, etc...) ou de savane à plantules fragiles (Ochno spp. Croton macrostachyus, Uapaca togoensis, Bridelio micronatha, Carissa edulis, Cussonia barteri, Nauclea latifolia, Ximenio americana, Vitex spp. Vernonia amygdalina, etc...).

Quant à l'ordre de fréquence des espèces, il. était et est devenu :

\begin{tabular}{|c|c|c|c|}
\hline & 1958 & 19 & \\
\hline aniellio oliveri... & & & \\
\hline a mocroptera & 2 & & \\
\hline Hottii ........ & 3 & & \\
\hline iymenocardia acida . & 4 & & \\
\hline naria............ & 5 & & \\
\hline gma thonningii $\ldots \ldots$ & 6 & & \\
\hline a lanceolata .......... & 7 & & \\
\hline chimperi ........... & 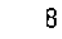 & & \\
\hline a madagascariensis & & & \\
\hline esa lanceolota & & & \\
\hline uclea latifolia. & & & \\
\hline
\end{tabular}

Cette évolution a été complétée bien entendu pendant ce laps de temps par un passage d'une partie des sujets dans la classe supérieure, ce qui ne fait qu'ajouter à l'épaisseur du couvert créé en 10 ans.

- Terrains basaltiques (R5).

L'ordre de fréquence des espèces est précisé dans le tableau ci-dessous pour les 3 inventaires. effectués.

\begin{tabular}{|c|c|c|c|}
\hline & 1958 & 1963 & 196 \\
\hline & & & \\
\hline Annona arenaria . ......... & 1 & 1 & I \\
\hline Piliostigma thanningil . . . . . . & 2 & 2 & 2 \\
\hline Hymenocardia acida ...... & 3 & 3 & 8 \\
\hline Syzygium guineense $\ldots . . .$. & 4 & 4 & 7 \\
\hline Psorospermum glaberrimum. . & 5 & 5 & 5 \\
\hline Trichilia roka $\ldots . . . \ldots \ldots$ & 6 & 7 & 10 \\
\hline Proté elliottil .......... & 7 & 6 & 12 \\
\hline Bridelia ferrugineo ........ & 8 & 9 & 14 \\
\hline Vitex madiensis $\ldots \ldots \ldots \ldots$ & 9 & 10 & \\
\hline Stereospermum kunthranum . . & 10 & - & \\
\hline Harungana madagascariensis & $\cdots$ & 8 & \\
\hline Allophylus africanus ........ & - & - & 4 \\
\hline Steganotaenia araliacea ..... & - & - & \\
\hline Ficus copensis . . . . . . . . & & & \\
\hline Maesa lanceolata . ......... & - & & \\
\hline
\end{tabular}

Ici encore, on constate un accroissementglobal du nombre des individus et un passage. de classe pour une partıe d'entre eux, mais le phénomène est devenu incontestablement plus lent qu'en terrains granitıques. En fait, partant d'un couvert arboré plus important en terrain granitique, Il était normal que le phénomène de reprise par la forêty soit plus rapide d'autant que. la présence constante de galeries contribue à. 
accroître l'ambiance forestière nécessaıre au départ de l'évolution.

Dans les terrains basaltiques, cette ambiance s'est créée plus lentement, là encore autour d'Harungana madagascariensis et d'Allophylus africanus formant ici aussı des fourrés épais renforcés d'espèces plus ou moins sarmenteuses (Cissus quadrangularis, Phyllonthus muellerionus, etc...) et protégeant des espèces d'installation relativement délicate (Steganotaenia araliacea, Ficus capensis, Fagara tessmanii, Sapium ellipticum, Vernonia amygdalina, etc...). Et maintenant le Moesa lanceolata vient renforcer l'ambiance. Dans 5 ans, il est probable que nous serons, d̀ des nuances spécifiques près, au stade actuel des terrains granitiques.

\section{$2^{\circ}$ Evolution sur l'ensemble des Parcs :}

L'évolution étudiée précédemment nous permet en considérant les tableaux II et II bis de constater qu'en moyenne tous les parcs évoluent à peu près dans le même sens d'un réembuissonnement avec à peu de choses près la même fluctuation de l'ordre des espèces et les mêmes apparitions.

Tous les parcs granitiques évolvent ef atteignent un fort degré de multiplication des sujets qui, de 1.000 à l'ha en 1958 , sont devenus 2.360 dix ans après. Sauf en G1 ef G6, l'Harungona madagascariensis a pris partout la première place. Pour les autres parcs ce n'est plus qu'une question de temps.

Pour la série basaltique rouge, l'embuissonnement s'est pratıquement maintenu passant de 815 sujets à l'ha à 790 entre 1958 et 1968.

Enfin, la série basaltique foncée montre une régression de 700 à 620 .

Il semble à priori que pour ces deux séries on retrouve encore l'ınfluence des possibilités effectives de charge ; mais surtout Il fout considérer que malgré l'évolution numérique globale, le couvert arboré s'est considérablement épaıssi partout par suite de l'accession générale à l'état d'arbrisseaux d'une bonne partie des jeunes sujets.

L'analyse spécifique montre aussı la même générale apparition des espèces colonisatrices.

Partis d'un niveau général plus bas d'embuissonnement les parcs basaltiques voient leur ambiance boisée s'installer plus lentement que pour les terrains granitiques mais inéluctablement.

\section{D) CONSÉQUENCES PRATIQUES}

\section{a) Débroussaillement :}

Nous avons en 1964 (8) exposé comment dans la méthode traditionnelle d'élevage foulbé, des mises en défens répétées et successives avec feux de pleine saison sèche pouvaient maintenir à un niveau convenable l'embuissonnement des pâturoges. Cette méthode comparable à la longue jachère agricole n'était cependant possible qu'à une époque ou les terrains de parcours étalent surabondants. Pour différentes raisons, ce temps est révolu mais hélas, l'élevage demeure au stade élémentaire de la cuelllette dont on veut tırer le maximum sans contrepartie. C'est dans cet esprit que les essass sur les feux ont été entrepris, mals là encore le travail «au minimum» ne permettra rien de bon. Notre première conclusion est que dès l'ınstant où l'on prétend exploiter un pâturage chaque saison des pluies (et c'est le cas actuel de tout l'Adamaoua, sauf dans les zones de transhumance), aucun régime de feux ne parviendra à contenir la végétation ligneuse. A vrai dire, et le chapitre suivant le montrera, la régression aveugle de l'élément arboré n'est pas -souhaitable tellement la pâture des espèces appétées peut avoir d'importance en salson sèche.

C'est donc l'homme encore qui, mécaniquement, maintiendra l'équilibre (le berger, s'il le veut, peut avoir tout au long de l'année un énorme pouvorr d'élimination des espèces indésırables). Aux moyens mécaniques ou manuels pourront s'ajouter l'empoisonnement des couches aux débroussaillants chimıques.

Nous rappelons que de même qu'il est nécessaire d'effectuer plusieurs coupes à la matchette pour nettoyer un pâturage, plusieurs traıtements hormonaux sont indispensables pour avoir des résultats durables (9).

La seconde conclusion est que les interventions mécaniques ne seront pas éternellement efficaces, car il existe un stade d'irréversibilité : c'est celul aù l'embuissonnement a atteınt une telle intensıté qu'il a réussi à éliminer les grandes espèces gramınéennes qui constituent habıtuellement la base du pâturage. Sans porte-graines, ces espèces mettront très longtemps à recoloniser le terrain. A ce stade d'évolution, l'élimination des arbres n'entraînera pas aussitôt une recons- 


\section{ANNEXE}

Noms scientifiques et vernaculaires (Boya) des espèces ligneuses de la station fourragère de Wakwa

(Savane et partie Galerie)

S : Savane
A : Appeté
TA : Très appeté
PA : Peu appeté
NA: Non appeté
$\mathrm{F}$ : Fréquent

TF : Très fréquent

PF : Peu fréquent

$\mathrm{R}$ : Rare

\begin{tabular}{|c|c|c|c|c|c|}
\hline Noms scientifiques & Famille & $\begin{array}{c}\text { Noms } \\
\text { vernaculares }\end{array}$ & Station & Appetab. & Fréq. \\
\hline 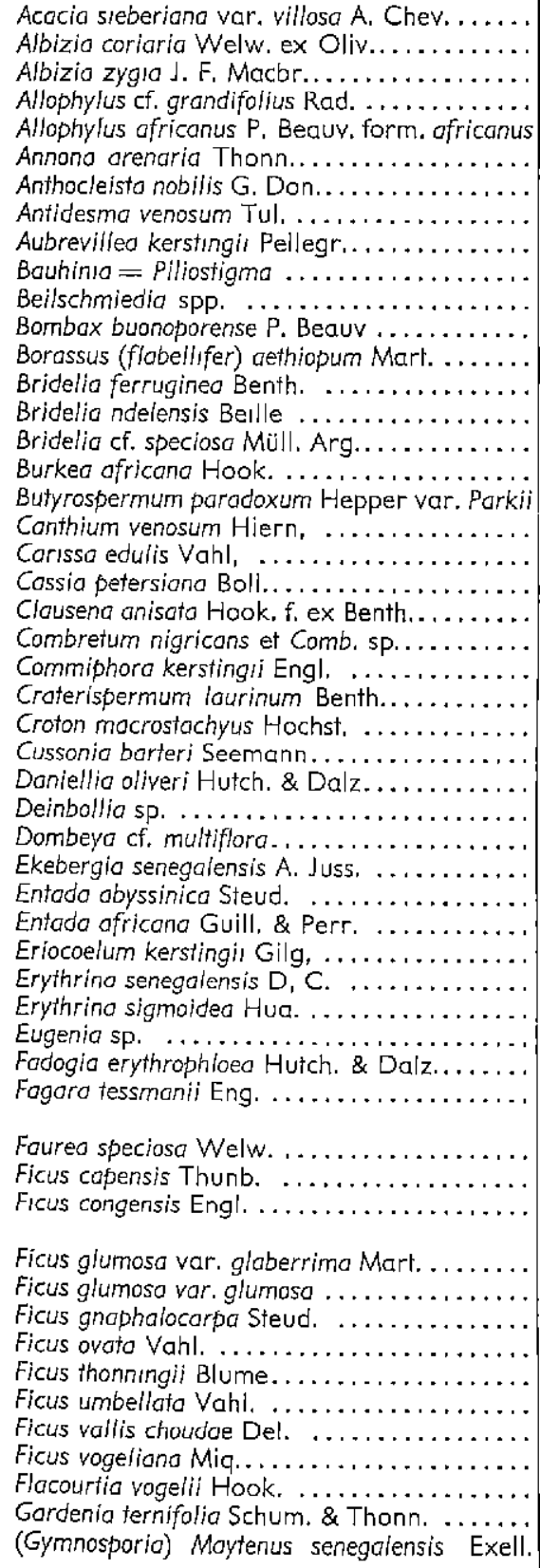 & $\begin{array}{l}\text { Mimosacées } \\
\text { Mimosacées } \\
\text { Mimosacées } \\
\text { Sapindacées } \\
\text { Sapındacées } \\
\text { Annonacées } \\
\text { Loganiacées } \\
\text { Euphorbiacées } \\
\text { Mimosacées } \\
\text { Cæasalpiniacées } \\
\text { Lauracées } \\
\text { Bombacacées } \\
\text { Arecacées } \\
\text { Euphorbiacées } \\
\text { Euphorbiacées } \\
\text { Euphorbiacées } \\
\text { Caesalpiniacées } \\
\text { Sapotacées } \\
\text { Rubiacées } \\
\text { Apocynacées } \\
\text { Caesalpiniacées } \\
\text { Rutacées } \\
\text { Combrefacées } \\
\text { Burseracées } \\
\text { Rubiacées } \\
\text { Euphorbiacées } \\
\text { Araliacées } \\
\text { Caesalpiniacées } \\
\text { Sapindacées } \\
\text { Sterculiacées } \\
\text { Meliacées } \\
\text { Mimasacées } \\
\text { Mimosacées } \\
\text { Sapindacées } \\
\text { Papillionacées } \\
\text { Papilionacées } \\
\text { Myrtacées } \\
\text { Rubiacées } \\
\text { Rutacées } \\
\text { Proteacées } \\
\text { Moracées } \\
\text { Moracées } \\
\text { Mlacourtiacées } \\
\text { Mubıacées } \\
\text { Celastracées } \\
\text { Moracées } \\
\text { Maracées } \\
\text { Moracées } \\
\text { Moracées } \\
\text { Maracées } \\
\text { Moracées } \\
\text { Fores }\end{array}$ & $\begin{array}{l}\text { Ngah } \\
\text { Tolla } \\
\text { Ndoya } \\
\text { Wi Norzer } \\
\text { Lossa } \\
\text { Soré } \\
\text { Zereforo } \\
\text { Boufibane } \\
\text { Soumbou } \\
\text { Domo } \\
\text { Ngala } \\
\text { Guerra } \\
\text { Koh } \\
\text { Sopo ou Nor } \\
\text { Norzer } \\
\text { Sopoli } \\
\text { Nbékéré } \\
\text { Kol } \\
\text { Ngazidila } \\
\text { Pinsela } \\
\text { Tefoto } \\
\text { Bathé } \\
\text { Pinsela } \\
\text { Foufauifou } \\
\text { Bogna } \\
\text { Kela ou Keha } \\
\text { Sakabo } \\
\text { Soyl ou Sori } \\
\text { Henga } \\
\text { Nde-Nde } \\
\text { Nde-Nde } \\
\text { Ngekéré } \\
\text { Wi Borondong } \\
\text { Borondong } \\
\text { Wi-Zomo } \\
\text { Wi Kobo } \\
\text { Sototo ou } \\
\text { Tekoua Ngamnu } \\
\text { Mbora I } \\
\text { Tourou ou } \\
\text { Kolo Batouj } \\
\text { Kolo } \\
\text { Mbora } \\
\text { Balioko } \\
\text { Tui } \\
\text { Tourou } \\
\text { Mboro I } \\
\text { Gouka } \\
\text { Bouigatha } \\
\text { Kiri } \\
\text { Babang } \\
\text { Solo }\end{array}$ & 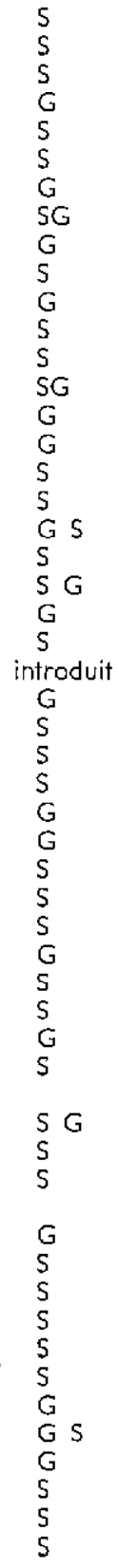 & $\begin{array}{l}\text { A } \\
\text { A } \\
\text { PA } \\
\text { TA }\end{array}$ & $\begin{array}{l}\mathrm{PF} \\
\mathrm{PF} \\
\mathrm{PF} \\
\mathrm{R} \\
\mathrm{PF} \\
\mathrm{TF} \\
\mathrm{PF} \\
\mathrm{PF} \\
\mathrm{PF} \\
\mathrm{TF} \\
\mathrm{R} \\
\mathrm{R} \\
\mathrm{PF} \\
\mathrm{F} \\
\mathrm{PF} \\
\mathrm{PF} \\
\mathrm{R} \\
\mathrm{PF} \\
\mathrm{PF} \\
\mathrm{PF} \\
\mathrm{R} \\
\mathrm{F} \\
\mathrm{F} \\
\mathrm{PF} \\
\mathrm{PF} \\
\mathrm{F} \\
\mathrm{TF} \\
\mathrm{R} \\
\mathrm{R} \\
\mathrm{R} \\
\mathrm{TF} \\
\mathrm{PF} \\
\mathrm{R} \\
\mathrm{R} \\
\mathrm{F} \\
\mathrm{R} \\
\mathrm{R} \\
\mathrm{F} \\
\mathrm{F} \\
\mathrm{PF} \\
\mathrm{PF} \\
\mathrm{PF} \\
\mathrm{PF} \\
\mathrm{PF} \\
\mathrm{PF} \\
\mathrm{F} \\
\mathrm{PF} \\
\mathrm{F} \\
\mathrm{PF} \\
\mathrm{P} \\
\mathrm{P} \\
\mathrm{P} \\
\end{array}$ \\
\hline
\end{tabular}




\begin{tabular}{|c|c|c|c|c|c|}
\hline Noms scientifiques & Fomille & $\begin{array}{c}\text { Noms } \\
\text { vernaculaires }\end{array}$ & Station & Appetab. & Freq. \\
\hline 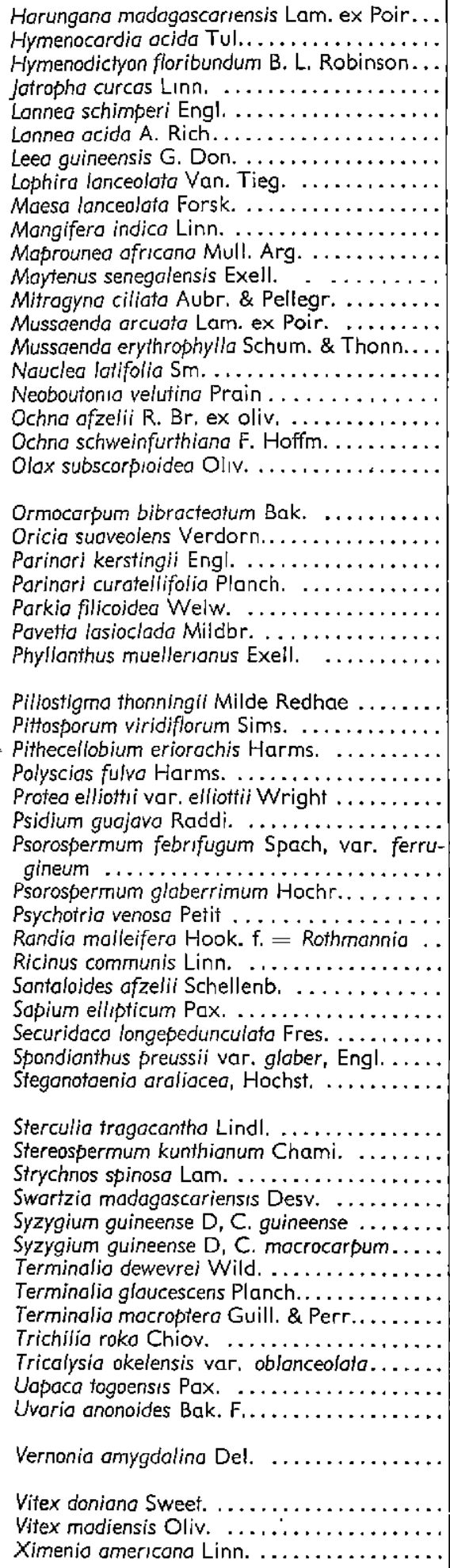 & 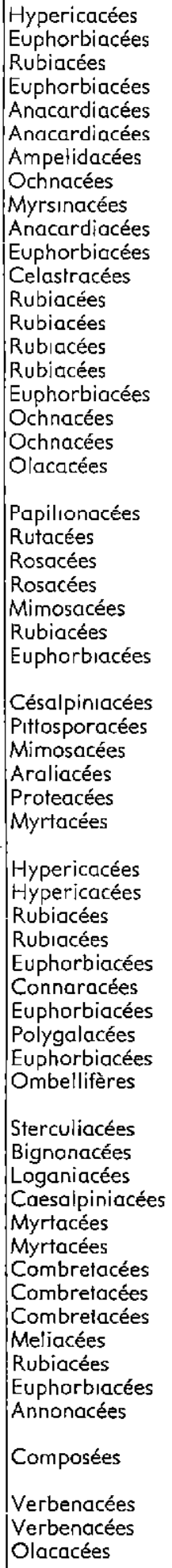 & 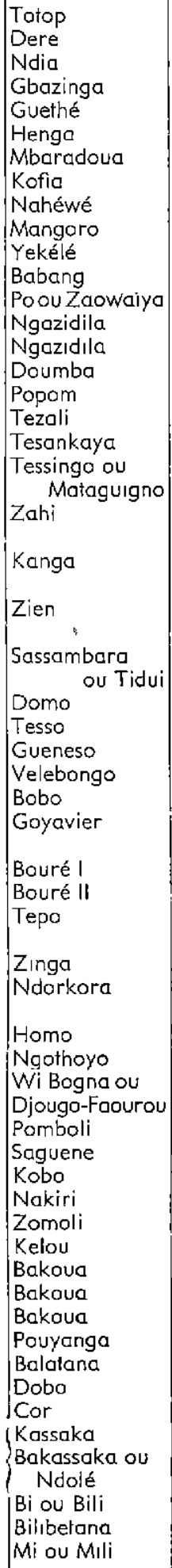 & 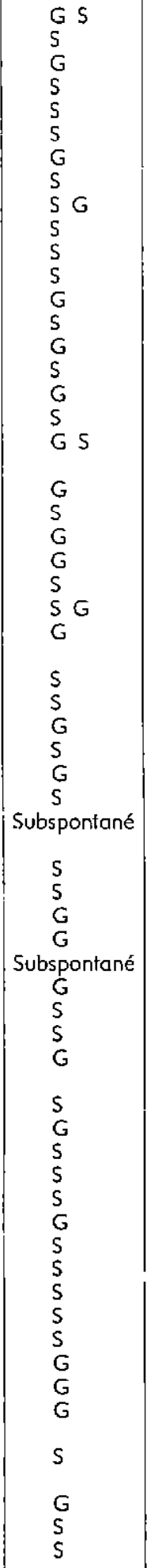 & $\begin{array}{l}\text { A } \\
\text { A } \\
\text { TA }\end{array}$ & $\begin{array}{l}\text { TF } \\
\text { TF } \\
\mathrm{PF} \\
\mathrm{PF} \\
\mathrm{F} \\
\mathrm{PF} \\
\mathrm{R} \\
\mathrm{F} \\
\mathrm{F} \\
\mathrm{F} \\
\mathrm{R} \\
\mathrm{F} \\
\mathrm{PF} \\
\mathrm{PF} \\
\mathrm{PF} \\
\mathrm{F} \\
\mathrm{PF} \\
\mathrm{PF} \\
\mathrm{PF} \\
\mathrm{F} \\
\mathrm{PF} \\
\mathrm{R} \\
\mathrm{PF} \\
\mathrm{PF} \\
\mathrm{PF} \\
\mathrm{PF} \\
\mathrm{TF} \\
\mathrm{PF} \\
\mathrm{R} \\
\mathrm{R} \\
\mathrm{TF} \\
\mathrm{PF} \\
\mathrm{F} \\
\mathrm{F} \\
\mathrm{F} \\
\mathrm{PF} \\
\mathrm{R} \\
\mathrm{F} \\
\mathrm{R} \\
\mathrm{PF} \\
\mathrm{R} \\
\mathrm{PF} \\
\mathrm{PF} \\
\mathrm{PF} \\
\mathrm{F} \\
\mathrm{R} \\
\mathrm{PF} \\
\mathrm{TF} \\
\mathrm{F} \\
\mathrm{PF} \\
\mathrm{TF} \\
\mathrm{PF} \\
\mathrm{F} \\
\mathrm{P} \\
\end{array}$ \\
\hline
\end{tabular}


titution du tapis herbacé cependant qu'elle livrera le sol aux brutalités de l'érosıon.

Des résultats obtenus, il semble bien qu'it soit souhaitable de laisser aux terrains granitiques à fort relief la vocation forestière qu'ils possèdent, cependant que sous cette forme, I's pourront tout de même fournir une pâture arborée si précieuse en saison sèche.

b) Régime des Feux :

Nous avons eu l'occasion (9) de traiter du problème des feux de brousse ef de montrer combien souvent on entamait un «dialogue de sourd » à ce sujet en ne distınguant pas d'entre les feux, ceux qui sont à usage de l'agriculteur, du pasteur, du forestier (lui-même différent suivant qu'il agit en protecteur de la forêt ou en aménagiste de zones cynégétiques).

Tous ces feux ont une justification commune : l'élimination à bon compte (d'efforts et d'argent) d'un matériel végétal devenu indésirable. Tous ces feux ont un regrettable caractère : ils sont incontrôlés ou insuffisamment contrôlés ce qui accroit le côté nuisible de leur action puisqu'ils détruisent plus que nécessaire.

En matière pastorale, l'énoncé d'une doctrine devient simple dès que l'on a constaté le peu d'action qu'il y avalt à attendre du feu sur les ligneux lorsque le pâturage a été correctement exploité en saison des pluies. Cette règle est alors la suivante : « Un terrain de parcours qui a été pâturé en saison des plures ne doit pas brûler pendant la saison sèche suivante. »

Sans doute, certains pâturages mal nettoyés devront être débarrassés de leurs encombrants refus. Pour ceux là, un feu dès le retour des pluies résoudra le problème et l'on sacrifiera à l'érosion hydrique une partie du sol bien que certaines salsons s'installent par des pluies suffisamment modérées pour ne pas déclencher un ruissellement trop grave et la perte des cendres.

Reste le cas des zones de mise en différé qui correspondent pratiquement $\mathrm{ICl}$ aux zones de transhumance. Il est nécessaire de brûler ces pâturages si l'on veut en tirer quelque partie en salson sèche. Là, un bon feu précoce assez fort pour détruire les chaumes conviendra parfaltement en permettant un regain correct, et en étant cependant assez offensıf pour le matériel ligneux.

Tous ces feux ne seront néanmoins qu'un pisaller, et Ils seront remplacés par d'autres technıques (Girobroyeur, faucheuse, etc...) dès que l'on en aura les moyens matériels et dès que ce ne sera plus économiquement aberrant.

\section{SUMMARY}

Woody plants and savanna pasfures of the Adamaoua in Cameroun

This study of shrubby and arboreus savannas of the plateau is actual on account of important problems of woody invasion on the pastures in the whole of Adamaova.

The author studies the three important points of this question :

10 fire action on the woody vegetation :

$2^{\circ}$ part of the stocking rate on the same vegetation ;

$3^{\circ}$ pastoral value of woody elements in the pasture grounds.

Conclusions are given in order to define a fire organization for the forests and the pastures. Basis of a sylvo-pastoral planing are also proposed with a view to the breeding evolution to less extensive forms.

\section{RESUMEN}

Vegetales leñosos y pastos de las sabanas del Adamaua en Camerún

Este estudio de las sabanas arbustivas y arborescentes que cubren la meseta es actual si se considera los importantes problemas causados por las invasiones leñosas en los pastos de Adamaua en general. 


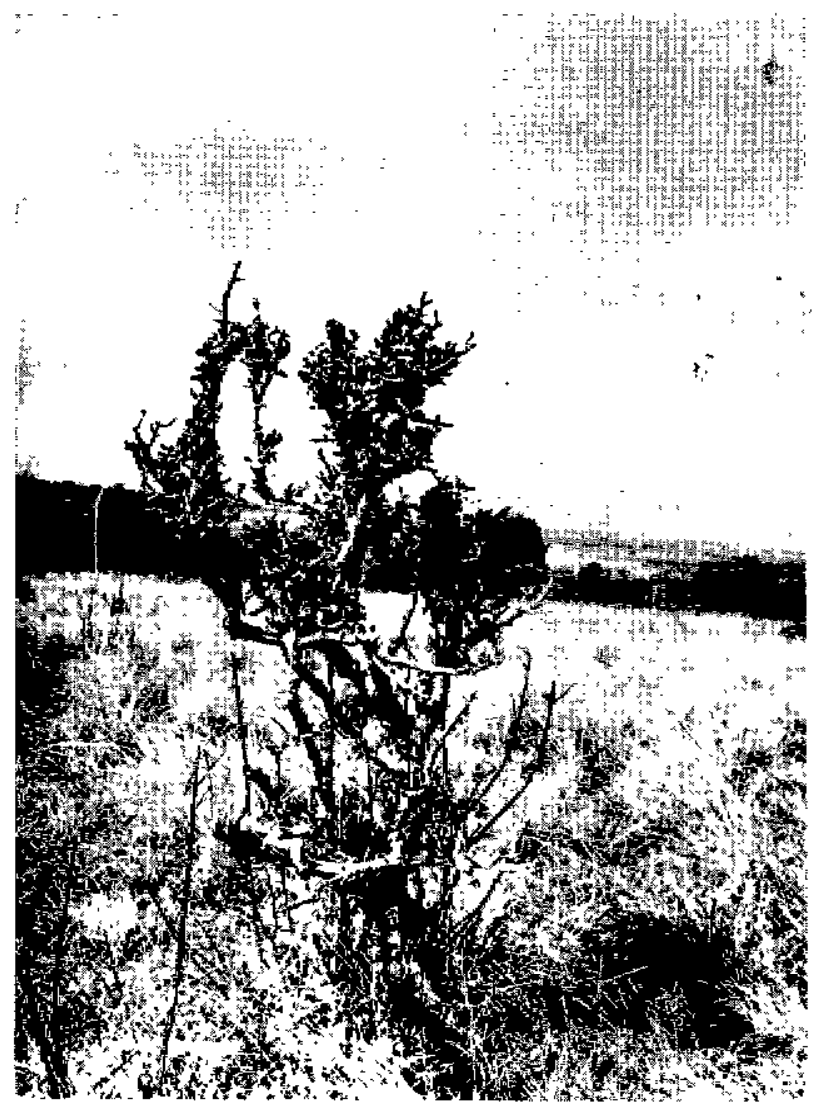

Fig. 1. - Les Gordenio sont ébourgeonnés au ras des rameaux.

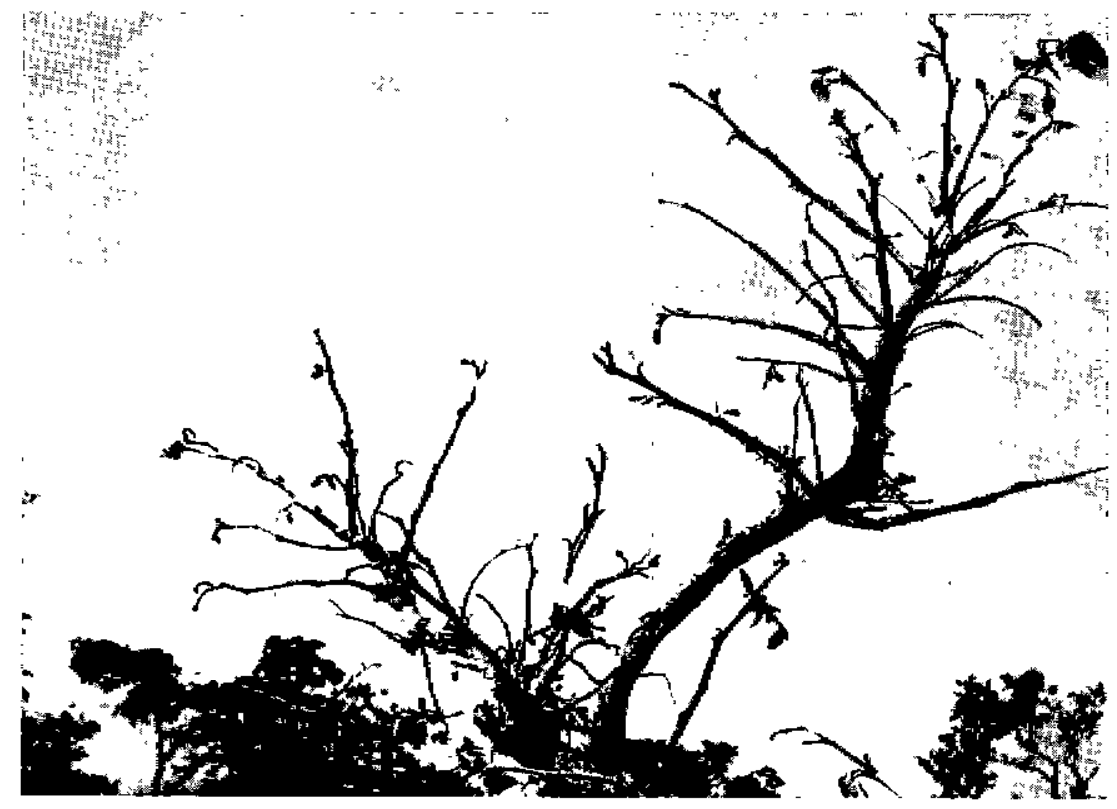

Fig. 2. - Rejet de Pliostigma. 


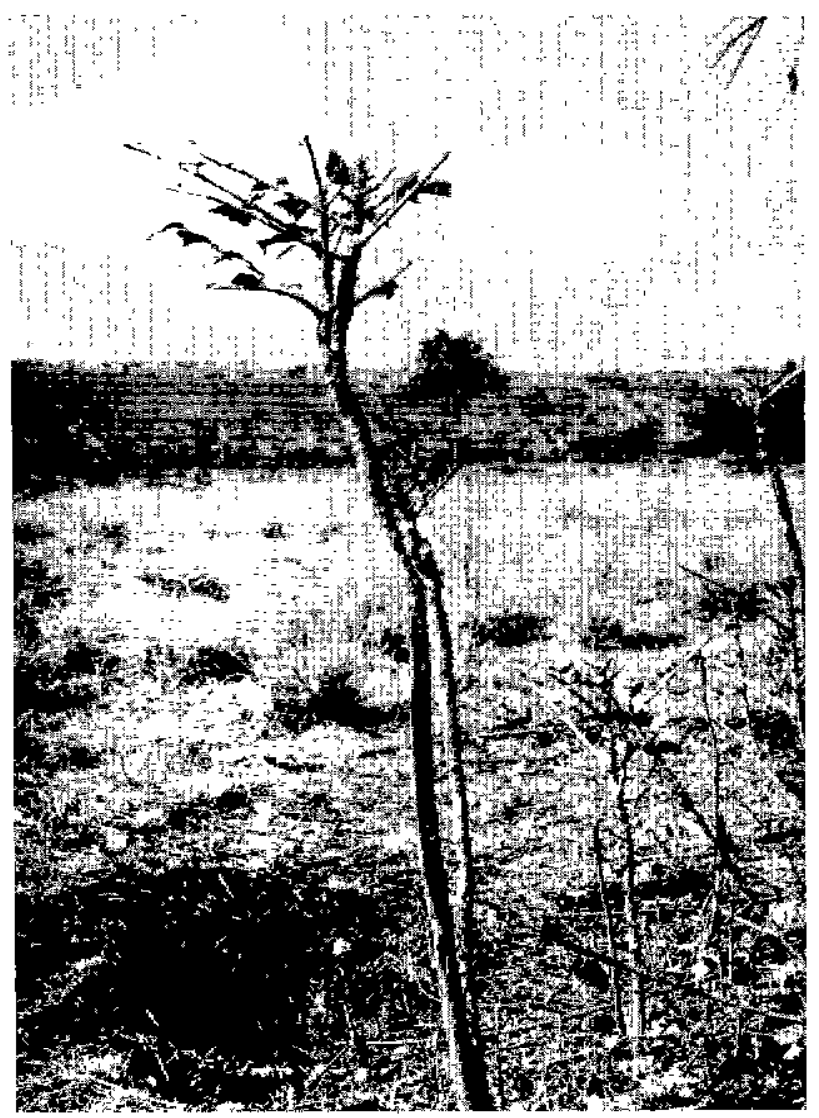

Fig. 3. - Ce jeune Fogara malgré ses épines est effeuillé et même écorcé, l'absence ou la faiblesse du feu lui permettra cependant de survivre.

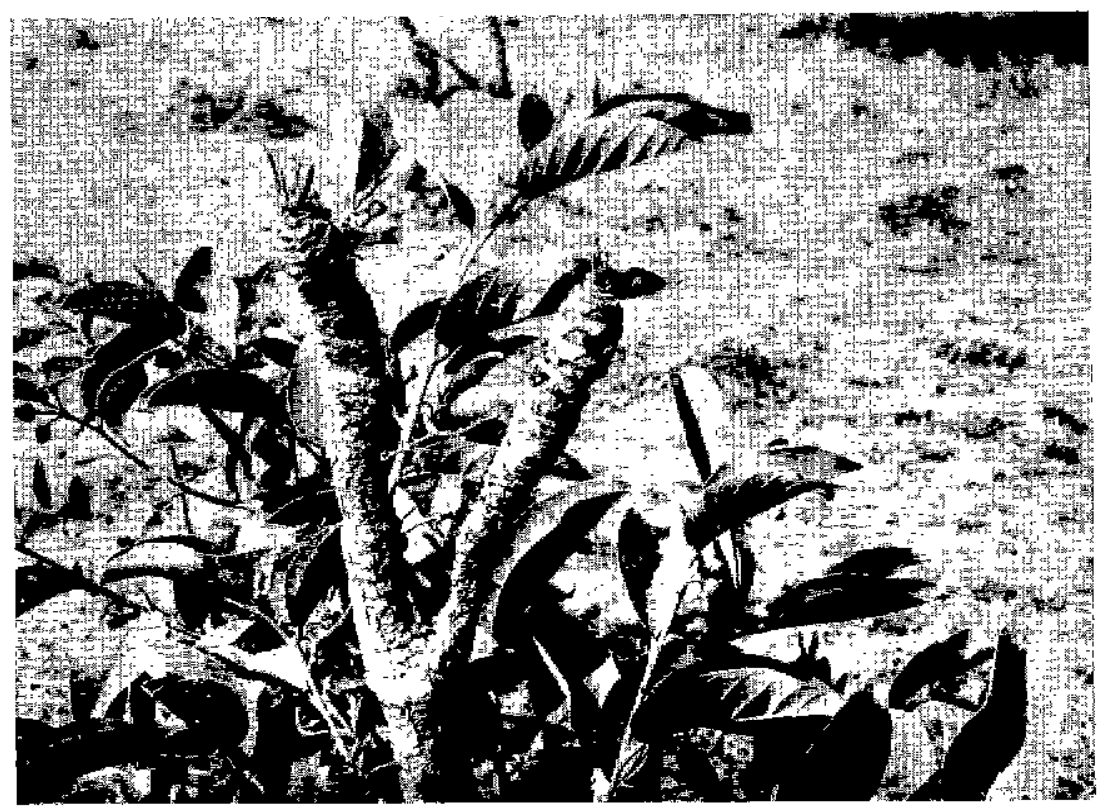

Fig. 4. - Ce jeune Cussonia ne fera pas une feuille intacte de toute la saison sèche l'Annona voisin restera intouché. 


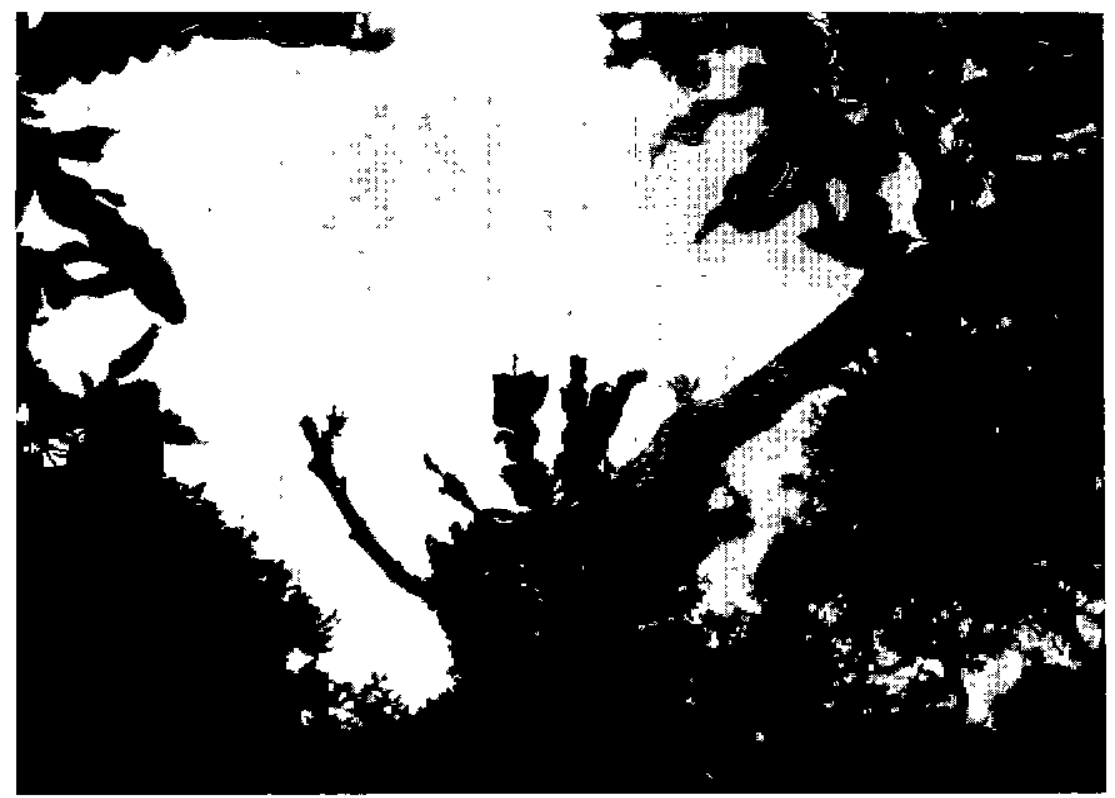

Fig. 5. - Les feuilles de Lophira deviennent vite coriaces et seules les extrémités sont arrachées.

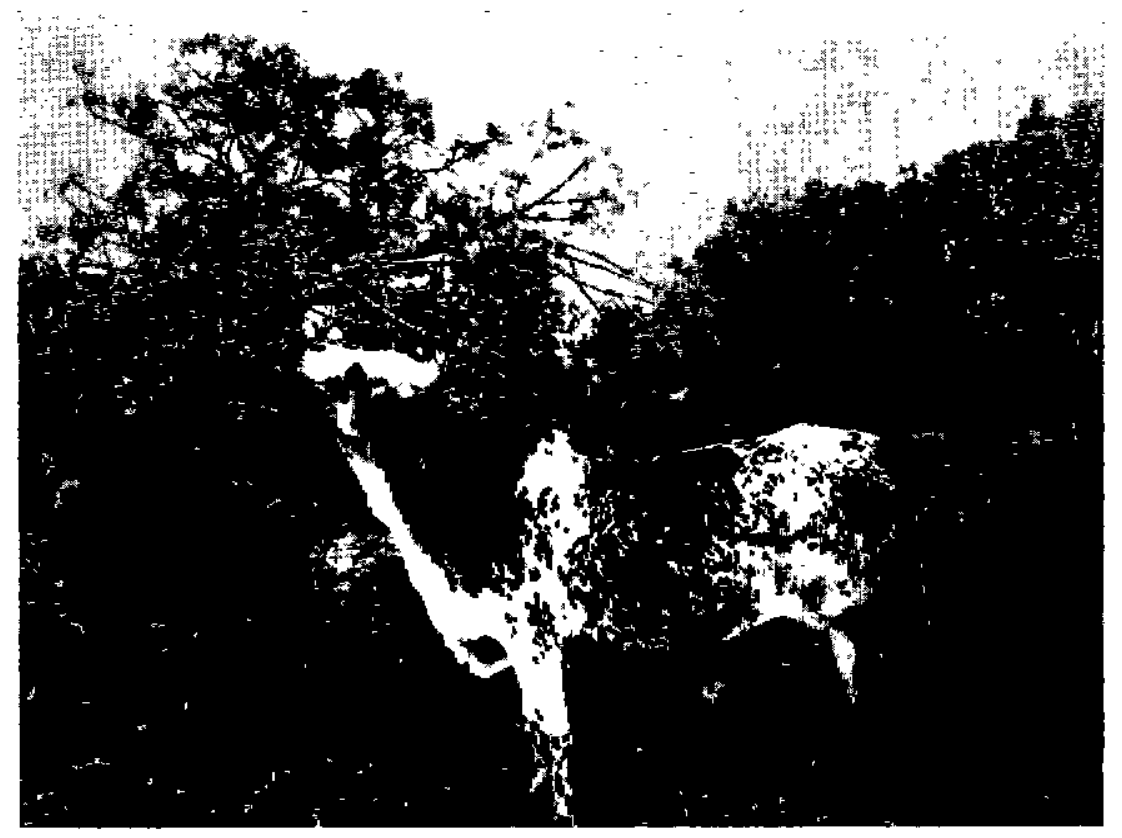

Fig. 6. - On se tordra le cou plus encore, mais tout ce que ce Lonnea peut offrir sera prélevé. 


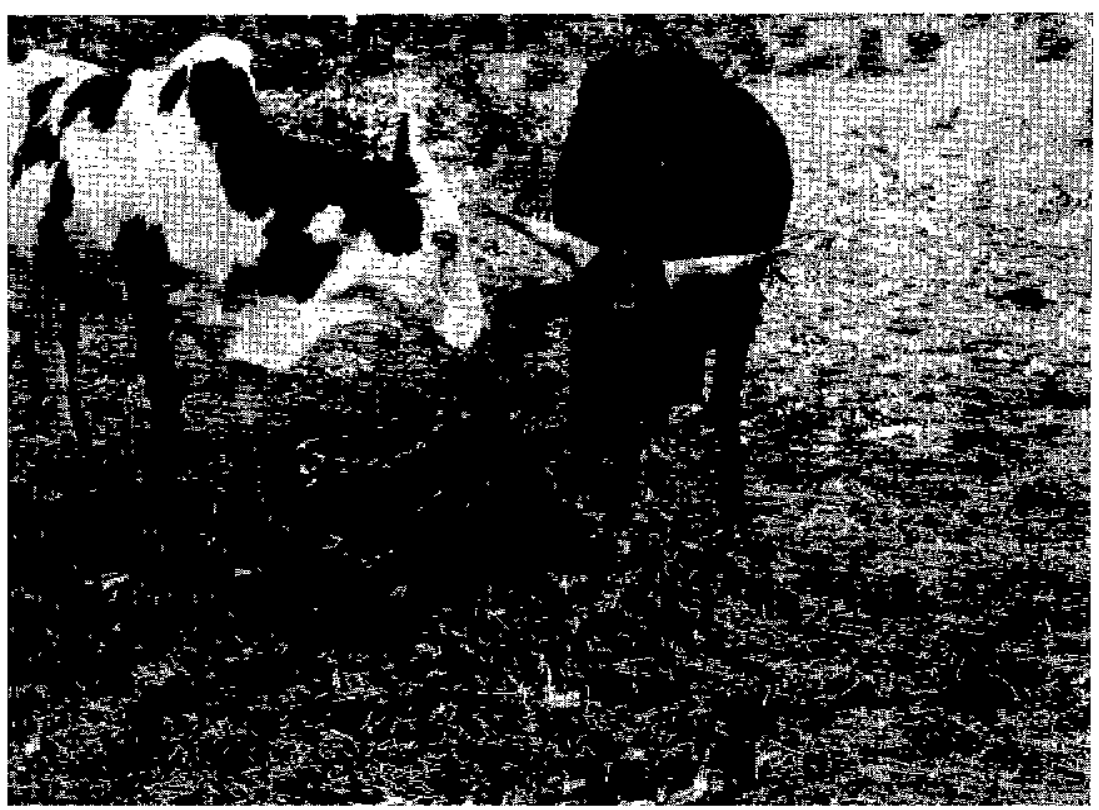

Fig. 7. - Sur cefte savane assez riche en espèces ligneuses, le bétail n'a rien d'autre à explotter. - Le tapis herbacé très ras en fin de saison des pluies a brûlé et le regain très modeste a disparu depuis longtemps.

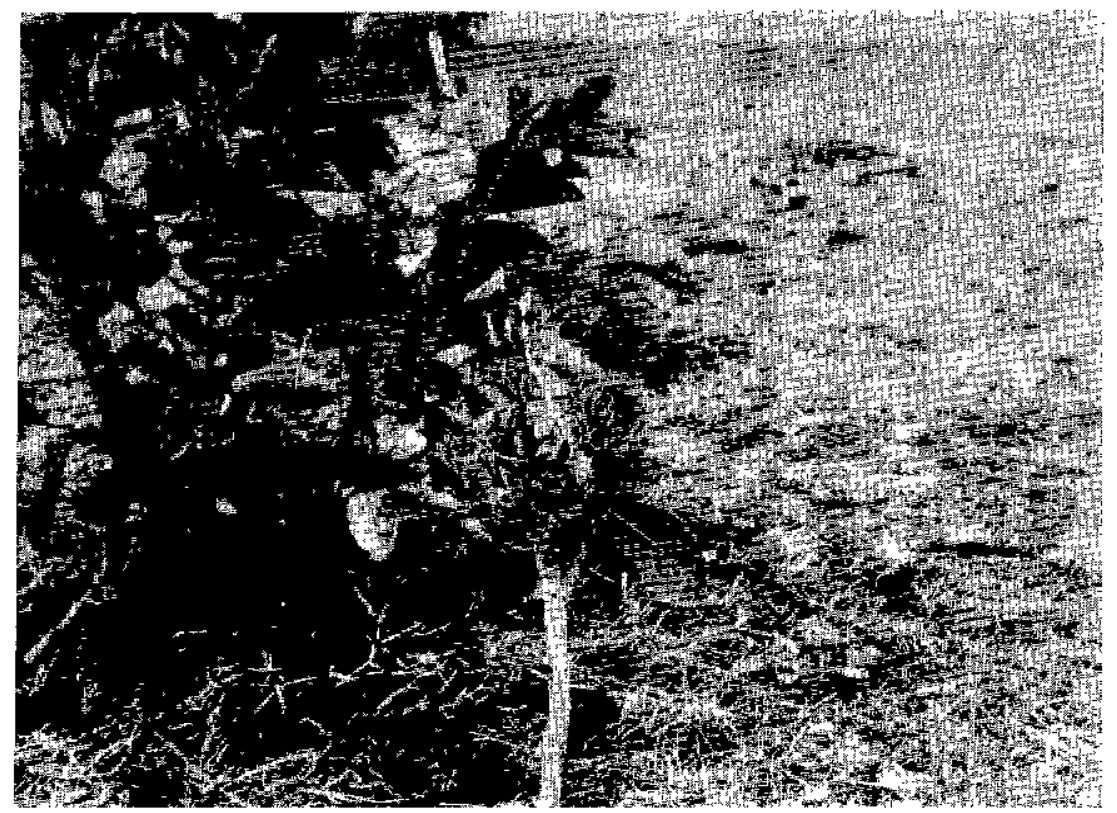

Fig. 8. - Peu de feuilles du Daniellia seront épargnées mais l'Annona derrière ne sera pas même effleuré. 
El autor estudia tres aspectos esenciales de esta vasta cuestión :

$1^{\circ}$ acción de los fuegos sobre la vegetación leñosa.

$2^{\circ}$ popel de la intensidad de la densidad de peso de ganado sobre dicha vegetación :

$3^{0}$ valor pastoral de los elementos leñosos de los terrenos de recorrido.

Se dan conclusıones tenıendo por objeto la definición de una politica de los fuegos en lo concerniente a las selvas y sobretodo los pastos. Se proponen tambien bases de instalación selva-pastoral con el fin de la evolución inevitable de la crianza hacia formas menos extensivas.

\section{BIBLIOGRAPHIE}

1. AUBREVILLE (A.), - Flore Forestière Soudano-Guinéenne. Paris, Soc. Ed. Géogr. Mar. et Col., 1950.

2. AUBREVILLE (A.), - Flore Forestière de Côte-d'lvoire (Publication no 15 du CTFT 1959).

2 bis. BILLE. - Expérimentation Agrosiologique en République Centrafricaine. IEMVT Étude agrostologique No 21.

3. BOUDET, - Etude du Ranch de Sipilou. Etude Agrostologique no 14 IEMVT, septembre 1966.

4. BOUDET et RIVIĖRE. - Emploi pratique des Analyses Fourragères pour l'appréciation des pâturages tropicaux. IEMVT Étude agrostologique no 8 juin 1967.
5. HUTCHINSON et DALZIEL. - Flora of West Tropical Afrika. Millbank; London, Crown agents for oversea gouvernments and administrations, 1954-1963.

6. MONNIER et PIOT. - Problèmes de Pâturages dans l'Adamaoua. Bols Forêts Trop., 1964 (97) : $3.15(98): 13-25$.

7. PICCIONI (M.). - Dictionnaire des Aliments pour les Animaux. Bologna, Edagricole, 1965.

8. PIOT. - Etudes Pastorales en Adamaoua Camerounais. Rev. Elev. Med. Vét. Pays trop. 1966, XIX (1) : 45-61.

9. PIOT. - Rapports Annuels Station Fourragère de WAKWA, 1965-66 et 1966-67.

10. SCHNEIDER. - Feeds of the World. Their digestibility and composition. Morgantown W. Virginio Agric. exper. Station, 1947. 